\title{
The parameters of Fibonacci and Lucas cubes*
}

\author{
Aleksandar Ilić \\ Facebook Inc, Menlo Park, CA, USA \\ Marko Milošević \\ Faculty of Sciences and Mathematics, University of Niš, Serbia
}

Received 10 August 2015, accepted 27 December 2015, published online 21 March 2016

\begin{abstract}
Motivated by the conjectures from Castro, et al. in 2011, in this paper we use integer programming formulations for computing the domination number, the 2-packing number and the independent domination number of Fibonacci cubes and Lucas cubes for $n \leq 13$.
\end{abstract}

Keywords: Fibonacci cubes, Lucas cubes, domination number, 2-packing number.

Math. Subj. Class.: 05C69, 05C25

\section{Introduction}

Hypercubes form one of the most applicable classes of graphs with many appealing properties. The $n$-cube $Q_{n}$ is the graph whose vertices are all binary strings of length $n$, and two vertices are adjacent if they differ in exactly one position. The Fibonacci cubes were introduced as a model for interconnection networks $[4,2]$. They offer challenging mathematical and computational problems, and admit a recursive decomposition into smaller Fibonacci cubes (see [5], [6], [8] for their structural properties). The Fibonacci cubes can be recognized in $O(m \log n)$ time (where $n$ is the order and $m$ the size of a given graph) [10]. The Lucas cubes [7] form a class of graphs closely related to the Fibonacci cubes, obtained by removing some vertices from the Fibonacci cubes.

Let $Q_{n}$ be the $n$-dimensional hypercube. A Fibonacci string of length $n$ is a binary string $b_{1} b_{2} \ldots b_{n}$ with $b_{i} \cdot b_{i+1}=0$ for $1 \leq i<n$. In other words, Fibonacci strings are binary strings that contain no consecutive ones. The Fibonacci cube $\Gamma_{n}$, for $n \geq 1$ is the subgraph of $Q_{n}$ induced by the Fibonacci strings of length $n$. A Fibonacci string $b_{1} b_{2} \ldots b_{n}$ is a Lucas string if $b_{1} \cdot b_{n}=0$. In other words, Lucas strings are binary strings

\footnotetext{
* This work was supported by Research Grants 174010 and 174033 of Serbian Ministry of Education and Science.

E-mail addresses: aleksandari@gmail.com (Aleksandar Ilić), marko643@gmail.com (Marko Milošević)
}

(a) (i) This work is licensed under http://creativecommons.org/licenses/by/3.0/ 
that contain no consecutive ones circularly. The Lucas cube $\Lambda_{n}$, for $n \geq 1$ is the subgraph of $Q_{n}$ induced by the Lucas strings of length $n$. It is well-known that $\left|V\left(\Gamma_{n}\right)\right|=F_{n+2}$, where $F_{n}$ are the Fibonacci numbers: $F_{0}=0, F_{1}=1, F_{n+1}=F_{n}+F_{n-1}$ for $n \geq 1$. Similarly, $\left|V\left(\Lambda_{n}\right)\right|=L_{n}$ for $n \geq 1$, where $L_{n}$ are the Lucas numbers: $L_{0}=2, L_{1}=1$, $L_{n+1}=L_{n}+L_{n-1}$ for $n \geq 1$.

Let $G$ be a graph. Set $D \subseteq V(G)$ is a dominating set if every vertex from $V(G)$ either belongs to $D$ or is adjacent to some vertex from $D$. The domination number $\gamma(G)$ is the minimum cardinality of a dominating set of $G$. A set $X \subseteq V(G)$ is called a 2-packing if $d(u, v)>2$ for any two different vertices $u$ and $v$ of $X$. The 2-packing number $\rho(G)$ is the maximum cardinality of a 2-packing of $G$. It is well-known that for any graph $G$ holds $\gamma(G) \geq \rho(G)$.

An independent set or stable set is a set of vertices in a graph, no two of which are adjacent. The independent domination number $i(G)$ of a graph $G$ is the size of the smallest independent dominating set (or, equivalently, the size of the smallest maximal independent set). The minimum dominating set in a graph will not necessarily be independent, but the size of a minimum dominating set is always less than or equal to the size of a minimum maximal independent set, $\gamma(G) \leq i(G)$.

Pike and Zou in [9] obtained a lower bound for the domination number of Fibonacci cube of order $n$ and determined the exact value of the domination number of Fibonacci cubes of order at most 8 . Castro et al. in [1] obtained upper and lower bounds for the domination and 2-packing number of Fibonacci and Lucas cubes. Furthermore, the authors obtained the exact values for $\gamma\left(\Gamma_{n}\right)$ and $\gamma\left(\Lambda_{n}\right)$ for $n \leq 9$ and for $\rho\left(\Gamma_{n}\right)$ and $\rho\left(\Lambda_{n}\right)$ for $n \leq 10$.

In this paper we use integer programming method to compute the exact values of the domination, 2-packing and independent domination number of Fibonacci and Lucas cubes for $n \leq 13$, which resolves the conjecture from [1].

\section{Main results}

For each subset of the vertex set $S \subseteq V(G)$ define

$$
x_{i}= \begin{cases}1 & \text { if } i \in S \\ 0 & \text { if } i \in V \backslash S .\end{cases}
$$

The neighborhood $N(v)$ of a vertex $v$ in a graph $G$ is the induced subgraph of $G$ consisting of all vertices adjacent to $v$ and all edges connecting two such vertices. Let $N[v]=N(v) \cup$ $\{v\}$ denote the closed neighborhood of the vertex $v$.

The domination number of $G$ can be formulated as the following $0-1$ integer programming problem:

$$
\gamma(G)=\min \sum_{i=1}^{n} x_{i}
$$

subject to

$$
\begin{gathered}
\sum_{j \in N[i]} x_{j} \geq 1, \\
x_{i} \in\{0,1\}, \quad \text { for all } 1 \leq i \leq n .
\end{gathered}
$$

It is easy to see that the conditions (2.2) and (2.3) define dominating set $S$ and vice versa [3]. For Fibonacci cube $\Gamma_{n}$ this formulation has $F_{n+2}$ variables and $2 F_{n+2}$ constraints, 
while each condition from (2.2) contains at most $n$ variables. For Lucas cube $\Lambda_{n}$ this formulation has $L_{n}$ variables and $2 L_{n}$ constrains, while each condition from (2.2) contains at most $n$ variables.

The 2-packing number of $G$ can be formulated as the following $0-1$ integer programming problem:

$$
\rho(G)=\max \sum_{i=1}^{n} x_{i}
$$

subject to

$$
\begin{gathered}
\sum_{j \in N[i]} x_{j} \leq 1, \\
x_{i} \in\{0,1\}, \quad \text { for all } 1 \leq i \leq n .
\end{gathered}
$$

We will prove that the conditions (2.5) and (2.6) define 2-packing set $S$ and vice versa. Let $S$ be a 2-packing set. Since $S$ does not contain two vertices on distance 1 or 2, for each $v \in V(G)$ there is at most one vertex from the closed neighborhood $N[v]$ which belongs to $S$. Assume now that the set $S$ satisfies the condition (2.5) and let $u$ and $v$ be two vertices from $S$ on distance 2 . In that case for the shortest path $v w u$, we have $\sum_{j \in N[w]} x_{j} \geq 2$, which is impossible. Therefore, $S$ is a 2-packing set.

The independent domination number $G$ can be formulated as the following $0-1$ integer programming problem:

$$
i(G)=\min \sum_{i=1}^{n} x_{i}
$$

subject to

$$
\begin{gathered}
\sum_{j \in N[i]} x_{j} \geq 1, \\
(n-1) x_{i}+\sum_{j \in N(i)} x_{j} \leq n-1, \\
x_{i} \in\{0,1\}, \quad \text { for all } 1 \leq i \leq n .
\end{gathered}
$$

The conditions (2.8) and (2.10) define domination set $S$, while the condition (2.9) ensures the independence. For $x_{i}=0$ we have always true $\sum_{j \in N(i)} x_{j} \leq n-1$, while for $x_{i}=1$ we have $\sum_{j \in N(i)} x_{j} \leq 0$ which is equivalent to $\sum_{j \in N[i]} x_{j}=1$. This proves that the formulation is correct. For Fibonacci cube $\Gamma_{n}$ this formulation has $F_{n+2}$ variables and $3 F_{n+2}$ constraints, while each conditions from (2.8) and (2.9) contain at most $n$ variables. For Lucas cube $\Lambda_{n}$ this formulation has $L_{n}$ variables and $3 L_{n}$ constrains, while each condition from (2.8) and (2.9) contain at most $n$ variables.

The tests were performed on the Intel Core 2 Duo T5800 $2.0 \mathrm{GHz}$ with 2 GB RAM running the Linux operating system and using CPLEX 8.1. The results are summarized in Tables 1 and 2. In Tables 3 and 4 we give some examples of dominating sets and 2-packing sets that were obtained during the computation of these values.

These results resolve the conjecture from [1] and support Problem 5.1 for $n \leq 12$. 


\begin{tabular}{r|rrrrrrrrrrr}
\hline$n$ & 1 & 2 & 3 & 4 & 5 & 6 & 7 & 8 & 9 & 10 & 11 \\
\hline$\left|V\left(\Gamma_{n}\right)\right|$ & 2 & 3 & 5 & 8 & 13 & 21 & 34 & 55 & 89 & 144 & 233 \\
$\left|E\left(\Gamma_{n}\right)\right|$ & 1 & 2 & 5 & 10 & 20 & 38 & 71 & 130 & 235 & 420 & 744 \\
$\gamma\left(\Gamma_{n}\right)$ & 1 & 1 & 2 & 3 & 4 & 5 & 8 & 12 & 17 & 25 & \\
$\rho\left(\Gamma_{n}\right)$ & 1 & 1 & 2 & 2 & 3 & 5 & 6 & 9 & 14 & 20 & 29 \\
$i\left(\Gamma_{n}\right)$ & 1 & 1 & 2 & 3 & 4 & 5 & 8 & 12 & 19 & 26 & \\
\hline
\end{tabular}

Table 1: Parameters of small Fibonacci cubes.

\begin{tabular}{r|rrrrrrrrrrrr}
\hline$n$ & 1 & 2 & 3 & 4 & 5 & 6 & 7 & 8 & 9 & 10 & 11 & 12 \\
\hline$\left|V\left(\Lambda_{n}\right)\right|$ & 1 & 3 & 4 & 7 & 11 & 18 & 29 & 47 & 76 & 123 & 199 & 322 \\
$\left|E\left(\Lambda_{n}\right)\right|$ & 0 & 2 & 3 & 8 & 15 & 30 & 56 & 104 & 189 & 340 & 605 & 1068 \\
$\gamma\left(\Lambda_{n}\right)$ & 1 & 1 & 1 & 3 & 4 & 5 & 7 & 11 & 16 & 23 & 35 & \\
$\rho\left(\Lambda_{n}\right)$ & 1 & 1 & 1 & 2 & 3 & 5 & 6 & 8 & 13 & 18 & 26 & 38 \\
$i\left(\Lambda_{n}\right)$ & 1 & 1 & 1 & 3 & 4 & 5 & 8 & 11 & 17 & 24 & 35 & \\
\hline
\end{tabular}

Table 2: Parameters of small Lucas cubes.

\begin{tabular}{|c|c|}
\hline \multicolumn{2}{|c|}{ Dominating set } \\
\hline$\Gamma(10)$ & $\Lambda(11)$ \\
\hline $\begin{array}{l}(0,1,0,1,0,0,0,0,0,0),(0,1,0,0,0,1,0,0,0,0) \\
(1,0,1,0,0,1,0,0,0,0),(1,0,0,1,0,0,1,0,0,0) \\
(0,0,0,0,1,0,1,0,0,0),(0,0,0,0,0,0,0,1,0,0) \\
(1,0,1,0,1,0,0,1,0,0),(1,0,0,1,0,1,0,1,0,0) \\
(1,0,0,0,0,0,0,0,1,0),(0,0,1,0,0,0,0,0,1,0) \\
(0,1,0,0,1,0,0,0,1,0),(0,0,0,1,0,1,0,0,1,0) \\
(0,0,1,0,0,0,1,0,1,0),(0,1,0,1,0,0,1,0,1,0) \\
(1,0,1,0,1,0,1,0,1,0),(0,0,0,1,0,0,0,0,0,1) \\
(1,0,0,0,1,0,0,0,0,1),(0,0,1,0,1,0,0,0,0,1) \\
(1,0,0,0,0,1,0,0,0,1),(0,1,0,0,0,0,1,0,0,1) \\
(1,0,1,0,0,0,1,0,0,1),(1,0,0,0,0,0,0,1,0,1) \\
(0,1,0,0,1,0,0,1,0,1),(0,0,1,0,0,1,0,1,0,1) \\
(0,1,0,1,0,1,0,1,0,1)\end{array}$ & $\begin{array}{l}(1,0,1,0,1,0,0,0,0,0,0),(1,0,0,0,0,1,0,0,0,0,0) \\
(0,1,0,0,0,0,1,0,0,0,0),(0,0,0,1,0,0,1,0,0,0,0) \\
(0,0,1,0,1,0,1,0,0,0,0),(0,0,0,0,0,0,0,1,0,0,0) \\
(1,0,1,0,1,0,0,1,0,0,0),(0,0,0,1,0,1,0,1,0,0,0) \\
(1,0,0,1,0,0,0,0,1,0,0),(0,0,0,0,1,0,0,0,1,0,0) \\
(0,0,1,0,0,1,0,0,1,0,0),(0,1,0,1,0,1,0,0,1,0,0) \\
(1,0,1,0,0,0,1,0,1,0,0),(0,0,0,0,1,0,1,0,1,0,0) \\
(0,0,1,0,0,0,0,0,0,1,0),(0,1,0,1,0,0,0,0,0,1,0) \\
(0,1,0,0,1,0,0,0,0,1,0),(0,0,0,1,0,1,0,0,0,1,0) \\
(1,0,0,0,0,0,1,0,0,1,0),(1,0,0,0,1,0,1,0,0,1,0) \\
(1,0,0,1,0,0,0,1,0,1,0),(0,0,0,0,1,0,0,1,0,1,0) \\
(0,1,0,0,0,1,0,1,0,1,0),(1,0,1,0,0,1,0,1,0,1,0) \\
(0,0,0,0,0,0,0,0,0,0,1),(0,0,1,0,1,0,0,0,0,0,1) \\
(0,1,0,0,0,1,0,0,0,0,1),(0,1,0,0,1,0,1,0,0,0,1) \\
(0,1,0,1,0,0,0,1,0,0,1),(0,1,0,0,1,0,0,1,0,0,1) \\
(0,0,1,0,0,1,0,1,0,0,1),(0,1,0,0,0,0,0,0,1,0,1) \\
(0,0,0,1,0,1,0,0,1,0,1),(0,0,1,0,0,0,1,0,1,0,1) \\
(0,1,0,1,0,0,1,0,1,0,1)\end{array}$ \\
\hline
\end{tabular}

Table 3: Examples of minimal dominating sets for $\Gamma(10)$ and $\Lambda(11)$

\begin{tabular}{l|l}
\hline \multicolumn{2}{c}{ 2-packaging set } \\
\hline$\Gamma(11)$ & $\Lambda(12)$ \\
\hline$(0,0,0,0,0,0,0,0,0,0,0),(1,0,1,0,0,0,0,1,0,0,0)$ & $(0,0,0,0,0,0,0,0,0,0,0,0),(1,0,0,1,0,1,0,0,0,0,0,0)$ \\
$(0,1,0,0,0,1,0,1,0,0,0),(0,1,0,0,1,0,0,0,1,0,0)$ & $(0,1,0,0,1,0,1,0,0,0,0,0),(1,0,1,0,1,0,0,1,0,0,0,0)$ \\
$(1,0,1,0,1,0,0,0,1,0,0),(0,0,0,1,0,1,0,0,1,0,0)$ & $(0,0,1,0,0,1,0,1,0,0,0,0),(1,0,0,0,1,0,0,0,1,0,0,0)$ \\
$(0,0,1,0,0,0,1,0,1,0,0),(0,1,0,1,0,0,1,0,1,0,0)$ & $(0,1,0,1,0,1,0,0,1,0,0,0),(1,0,1,0,0,0,1,0,1,0,0,0)$ \\
$(1,0,0,0,1,0,0,0,0,1,0),(0,0,1,0,0,1,0,0,0,1,0)$ & $(0,0,0,1,0,0,1,0,1,0,0,0),(1,0,1,0,0,0,0,0,0,1,0,0)$ \\
$(0,1,0,1,0,1,0,0,0,1,0),(0,1,0,0,0,0,1,0,0,1,0)$ & $(0,1,0,0,0,1,0,0,0,1,0,0),(1,0,0,1,0,0,1,0,0,1,0,0)$ \\
$(1,0,0,1,0,0,1,0,0,1,0),(0,0,1,0,1,0,1,0,0,1,0)$ & $(0,0,1,0,1,0,1,0,0,1,0,0),(0,1,0,1,0,0,0,1,0,1,0,0)$ \\
$(0,0,0,1,0,0,0,1,0,1,0),(0,1,0,0,1,0,0,1,0,1,0)$ & $(0,0,0,0,1,0,0,1,0,1,0,0),(1,0,0,0,0,1,0,1,0,1,0,0)$ \\
$(1,0,0,0,0,1,0,1,0,1,0),(0,0,1,0,1,0,0,0,0,0,1)$ & $(0,1,0,1,0,0,0,0,0,0,1,0),(1,0,1,0,0,1,0,0,0,0,1,0)$ \\
$(1,0,1,0,0,0,1,0,0,0,1),(0,0,0,1,0,0,1,0,0,0,1)$ & $(0,0,1,0,0,0,1,0,0,0,1,0),(1,0,0,0,1,0,1,0,0,0,1,0)$ \\
$(0,1,0,0,1,0,1,0,0,0,1),(0,1,0,1,0,0,0,1,0,0,1)$ & $(1,0,0,0,0,0,0,1,0,0,1,0),(0,1,0,0,1,0,0,1,0,0,1,0)$ \\
$(1,0,0,0,1,0,0,1,0,0,1),(0,0,1,0,0,1,0,1,0,0,1)$ & $(0,0,0,1,0,1,0,1,0,0,1,0),(1,0,0,1,0,0,0,0,1,0,1,0)$ \\
$(1,0,0,1,0,1,0,1,0,0,1),(1,0,0,1,0,0,0,0,1,0,1)$ & $(0,0,1,0,1,0,0,0,1,0,1,0),(0,0,0,0,0,1,0,0,1,0,1,0)$ \\
$(0,1,0,0,0,1,0,0,1,0,1),(1,0,1,0,0,1,0,0,1,0,1)$ & $(0,1,0,0,0,0,1,0,1,0,1,0),(0,0,1,0,1,0,0,0,0,0,0,1)$ \\
$(1,0,0,0,1,0,1,0,1,0,1)$ & $(0,1,0,1,0,0,1,0,0,0,0,1),(0,0,0,1,0,0,0,1,0,0,0,1)$ \\
& $(0,1,0,0,0,1,0,1,0,0,0,1),(0,1,0,0,0,0,0,0,1,0,0,1)$ \\
& $(0,0,1,0,0,1,0,0,1,0,0,1),(0,0,0,0,1,0,1,0,1,0,0,1)$ \\
& $(0,1,0,0,1,0,0,0,0,1,0,1),(0,0,0,1,0,1,0,0,0,1,0,1)$ \\
& $(0,0,0,0,0,0,1,0,0,1,0,1),(0,0,1,0,0,0,0,1,0,1,0,1)$ \\
\hline
\end{tabular}




\section{References}

[1] A. Castro, S. Klavžar, M. Mollard and Y. Rho, On the domination number and the 2-packing number of Fibonacci cubes and Lucas cubes, Comput. Math. Appl. 61 (2011), 2655-2660, doi:10.1016/j.camwa.2011.03.012, http://dx.doi.org/10.1016/j.camwa.2011. 03.012 .

[2] P. Gregor, Recursive fault-tolerance of Fibonacci cube in hypercubes, Discrete Math. 306 (2006), 1327-1341, doi:10.1016/j.disc.2004.09.017, http://dx.doi.org/10.1016/ j.disc.2004.09.017.

[3] T. W. Haynes, S. T. Hedetniemi and P. J. Slater, Fundamentals of domination in graphs, volume 208 of Monographs and Textbooks in Pure and Applied Mathematics, Marcel Dekker, Inc., New York, 1998.

[4] W.-J. Hsu, Fibonacci cubes-a new interconnection topology, IEEE Transactions on Parallel and Distributed Systems 4 (1993), 3-12, doi:10.1109/71.205649.

[5] A. Ilić, S. Klavžar and Y. Rho, Generalized Fibonacci cubes, Discrete Math. 312 (2012), 2-11, doi:10.1016/j.disc.2011.02.015, http://dx.doi.org/10.1016/j.disc.2011.02. 015.

[6] S. Klavžar, On median nature and enumerative properties of Fibonacci-like cubes, Discrete Math. 299 (2005), 145-153, doi:10.1016/j.disc.2004.02.023, http://dx.doi.org/ 10 . $1016 / j . d i s c .2004 .02 .023$.

[7] E. Munarini, C. Perelli Cippo and N. Zagaglia Salvi, On the Lucas cubes, Fibonacci Quart. 39 (2001), 12-21.

[8] E. Munarini and N. Z. Salvi, Structural and enumerative properties of the Fibonacci cubes, Discrete Math. 255 (2002), 317-324, doi:10.1016/S0012-365X(01)00407-1, Combinatorics '98 (Palermo), http://dx.doi.org/10.1016/s0012-365x (01)00407-1.

[9] D. A. Pike and Y. Zou, The domination number of Fibonacci cubes, J. Combin. Math. Combin. Comput. 80 (2012), 433-444.

[10] A. Taranenko and A. Vesel, Fast recognition of Fibonacci cubes, Algorithmica 49 (2007), 81-93, doi:10.1007/s00453-007-9026-5, http://dx.doi.org/10.1007/ s $00453-007-9026-5$. 